\title{
THE APPELL TRANSFORM AND THE SEMIGROUP PROPERTY FOR TEMPERATURES
}

\author{
ELIZABETH KOCHNEFF and YORAM SAGHER
}

(Received 24 January 1994)

Communicated by P. C. Fenton

\begin{abstract}
We prove that if $u(x, t)$ is a solution of the one dimensional heat equation and if $\mathscr{A} u(x, t)$ is its Appell transform, then $u(x, t)$ has the semi-group (Huygens) property in a domain $D$ if and only if $\mathscr{A} u(x, t)$ has the semi-group property in a dual region. We apply this result to simplify and extend some results of Rosenbloom and Widder.
\end{abstract}

1991 Mathematics subject classification (Amer. Math. Soc.): 35K05, 42 C99.

\section{Introduction}

There are remarkable parallels between the behavior of some solutions of the heat equation in one space variable and harmonic and analytic functions. These parallels clearly carry the investigation of solutions of the heat equation to situations which cannot be realized in a physical sense; however, the same is true of analytic function theory. We refer the reader to [3] where an extensive list of analogies between analytic functions and solutions of the heat equation is given.

One important feature of analytic function theory is the incorporation of the point at infinity. It gives a systematic treatment of a number of important results which could, however, be stated and proved without reference to the point at infinity.

The complex plane with the point at infinity incorporated is visualized using the Riemann sphere. The corresponding construction for the heat equation consists of wrapping the $(x, t)$-plane into a cylinder which we propose to call the Appell cylinder. The role of the south pole of the Riemann sphere is taken by the line $\{(x, 0): x \in R\}$. The role of the north pole is taken by the line $\{(x, \infty)\}$.

The point at infinity in analytic function theory is handled by the transformation

(C) 1996 Australian Mathematical Society 0263-6115/95 \$A2.00+0.00 
$f(z) \rightarrow f(1 / z) / z$. The line at infinity for temperature functions is similarly handled by the Appell transform, which is defined for $t \neq 0$ by [1]:

$$
\mathscr{A} u(x, t)=k(x, t) u\left(\frac{x}{t}, \frac{-1}{t}\right)
$$

where

$$
k(x, t)=\frac{1}{\sqrt{4 \pi t}} e^{-x^{2} / 4 t}
$$

is the heat kernel.

If $u(x, t)$ satisfies the heat equation on $(-\infty, \infty) \times I, I=(a, b)$, then $\mathscr{A} u(x, t)$ satisfies the heat equation in $(-\infty, \infty) \times \tilde{I}$ where

$$
\tilde{I}=\{t:-1 / t \in I\} \text {. }
$$

The structure of the Appell cylinder changes our notions of time intervals and linear ordering. We will denote by $(a, b)$ when $a<b$ (an interval of the first kind) the set of all $a<t<b$. When $b<0<a,(a, b)$ (an interval of the second kind) will denote the complement of $[b, a]$ (including $t=\infty$ ).

If $I$ is of the first kind and $a<t_{1}, t_{2}<b$ then we say $t_{1}<_{I} t_{2}$ if $t_{1}<t_{2}$. If $I$ is of the second kind, then we say $t_{1}<_{1} t_{2}$ if either $t_{1}<t_{2}<b, a<t_{1}<t_{2}$ or both $t_{1}>a$ and $t_{2}<b$. It can be seen that the new order is consistent with the Appell transform in the following sense: $t<_{I} s$ if and only if $-1 / t<_{I}-1 / s$.

Observe that if $I$ is of the first kind and contains $t=0$, then $\tilde{I}$ is of the second kind and vice versa. Observe also that $(\widetilde{\tilde{I}})=I$.

DEFINITION 1. A solution of the heat equation $u(x, t)$ satisfies the semi-group (Huygens) property in $I=(a, b)$ if and only if for $-\infty<x<\infty, a<_{I} t^{\prime}<_{I} t<_{1} b$ we have

$$
u(x, t)=\int_{R} k\left(x-y, t-t^{\prime}\right) u\left(y, t^{\prime}\right) d y .
$$

We then write $u(x, t) \in H^{*}(I)$.

If $I$ is an interval of the first kind, this definition coincides with the usual one, see for example [2].

We will show that there exist solutions of the heat equation which satisfy the semigroup property in time intervals of the second kind. This corresponds to functions which are analytic or harmonic in $\{z:|z|>r\}$. 
A first example of this phenomenon follows from a reinterpretation of a calculation of Rosenbloom and Widder (see [2]). They showed that if $1-4 A t>0$, then we have

$$
\int_{R} k(x-y, t) e^{A y^{2}} d y=\frac{e^{A x^{2} /(1-4 A t)}}{(1-4 A t)^{1 / 2}} .
$$

Their interpretation of this calculation was to give an example of an initial temperature distribution for which the semi-group property holds for a limited time only: $0<t<1 / 4 A$. However, if we take $A=-1 / 4 s$ with $s$ negative we can view their result as a manifestation of the semi-group property for the heat kernel with negative times. With this interpretation we observe that the condition $1-4 A t>0$ prevents us from exiting the time interval of the second type $(0+, 0-)$.

We will get the Rosenbloom-Widder result, (5), as an immediate corollary of Theorem 1 below.

\section{The Appell transform and the semi-group property}

THEOREM 1. If

$$
u(x, t) \in H^{*}(I)
$$

then

$$
\mathscr{A} u(x, t) \in H^{*}(\tilde{I}) .
$$

PROOF. Since $u(x, t) \in H^{*}(I)$, we have

$$
u(x, t)=\int_{R} k\left(x-y, t-t^{\prime}\right) u\left(y, t^{\prime}\right) d y
$$

whenever $a<_{I} t^{\prime}<_{I} t<_{I} b$. Therefore, if $\tau, \tau^{\prime} \neq 0$ we have for $a<_{I}-1 / \tau^{\prime}<_{I}$ $-1 / \tau<{ }_{1} b$,

$$
\begin{aligned}
\mathscr{A} u(x, \tau) & =k(x, \tau) \int_{R} k\left(\frac{x}{\tau}-y, \frac{-1}{\tau}-t^{\prime}\right) u\left(y, t^{\prime}\right) d y \\
& =k(x, \tau) \int_{R} k\left(\frac{x}{\tau}-y, \frac{-1}{\tau}+\frac{1}{\tau^{\prime}}\right) u\left(y, \frac{-1}{\tau^{\prime}}\right) d y \\
& =k(x, \tau) \int_{R} k\left(\frac{x}{\tau}-\frac{y}{\tau^{\prime}}, \frac{-1}{\tau}+\frac{1}{\tau^{\prime}}\right) u\left(\frac{y}{\tau^{\prime}}, \frac{-1}{\tau^{\prime}}\right) \frac{d y}{\tau^{\prime}} \\
& =\frac{k(x, \tau)}{\tau^{\prime}} \int_{R} k\left(\frac{x \tau^{\prime}-y \tau}{\tau \tau^{\prime}}, \frac{\tau-\tau^{\prime}}{\tau \tau^{\prime}}\right) \frac{1}{k\left(y, \tau^{\prime}\right)} \mathscr{A} u\left(y, \tau^{\prime}\right) d y \\
& =\int_{R} k\left(x-y, \tau-\tau^{\prime}\right) \mathscr{A} u\left(y, \tau^{\prime}\right) d y .
\end{aligned}
$$


Therefore since

$$
a<_{I} \frac{-1}{\tau^{\prime}}<_{I} \frac{-1}{\tau}<_{I} b
$$

is equivalent to

$$
\frac{-1}{a}<_{\tilde{I}} \tau^{\prime}<_{I} \tau<_{\tilde{I}} \frac{-1}{b},
$$

the theorem is proved except in the case $\tau, \tau^{\prime}=0$. In these cases the theorem follows from continuity and the dominated convergence theorem.

Since $1 \in H^{*}(-\infty, \infty)$, Theorem 1 shows that

$$
k(x, t)=\int_{R} k\left(x-y, t-t^{\prime}\right) k\left(y, t^{\prime}\right) d y
$$

whenever $0<t^{\prime}<t, t^{\prime}<t<0$ or both $t^{\prime}>0$ and $t<0$. This result implies (5).

A simple calculation shows:

THEOREM 2. For $t \neq 0$, whenever either integral exists,

$$
\int_{R} \mathscr{A} u(x,-t) \mathscr{A} v(x, t) d x=\frac{1}{4 \pi i} \int_{R} u\left(-x, \frac{1}{t}\right) v\left(x, \frac{-1}{t}\right) d x .
$$

We will show that Theorems 1 and 2 enable us to simplify and extend several results of [2].

For $n=0,1, \ldots$, the heat polynomials of degree $n$ are the unique polynomials of degree $n$ which satisfy the heat equation, have coefficient of $x^{n}$ equal to unity, and have homogeneity $n$. They are defined [2]:

$$
v_{n}(x, t)=n ! \sum_{k=0}^{[n / 2]} \frac{t^{k} x^{n-2 k}}{k !(n-2 k) !} .
$$

For $n=0,1, \ldots$, define the $n^{t h}$ Hermite polynomial with respect to the measure $e^{-x^{2} / 2} d x$ as:

$$
H_{n}(x)=(-1)^{n} e^{x^{2} / 2} \frac{d^{n}}{d x^{n}} e^{-x^{2} / 2}
$$

Then we have [2]:

$$
v_{n}(x, t)=(-2 t)^{n / 2} H_{n}\left(\frac{x}{\sqrt{-2 t}}\right)
$$


The associated heat polynomials given in [2] are defined for $n=0,1, \ldots$, as:

$$
w_{n}(x, t)=\mathscr{A} v_{n}(x, t)=\frac{1}{\sqrt{4 \pi t}} e^{-x^{2} / 4 t}\left(\frac{2}{t}\right)^{n / 2} H_{n}\left(\frac{x}{\sqrt{2 t}}\right) .
$$

The sequences $\left\{v_{n}\right\}$ and $\left\{w_{n}\right\}$ form a bi-orthogonal system. For $t>0$ :

$$
\int_{R} v_{m}(x,-t) w_{n}(x, t) d x=\delta_{m n}
$$

Just as $\sum a_{n} z^{n}$ are natural for the domain $|z|<r, \sum a_{n} v_{n}$ are natural for the domain $|t|<\sigma$ :

THEOREM 3. (Rosenbloom and Widder [2]) A necessary and sufficient condition that $u(x, t) \in H^{*}(-\sigma, \sigma)$ is that

$$
u(x, t)=\sum_{n=0}^{\infty} a_{n} v_{n}(x, t), \quad|t|<\sigma,
$$

where the convergence is pointwise. The coefficients $a_{n}$ are then given by:

$$
a_{n}=\frac{1}{n ! 2^{n}} \int_{R} u(y,-t) w_{n}(y, t) d y, \quad 0<t<\sigma .
$$

For expansions in terms of the $w_{n}$, Rosenbloom and Widder proved:

THEOREM 4. (Rosenbloom and Widder [2]) A necessary and sufficient condition that $u(x, t) \in H^{*}(\sigma, \infty)$ and that

$$
\int_{R}|u(x, t)| e^{x^{2} / 8 t} d x<\infty, \quad t>\sigma
$$

is that

$$
u(x, t)=\sum_{n=0}^{\infty} b_{n} w_{n}(x, t), \quad t>\sigma,
$$

where the convergence is pointwise. The coefficients $b_{n}$ are given by:

$$
b_{n}=\frac{1}{n ! 2^{n}} \int_{R} u(y, t) v_{n}(y,-t) d y, \quad t>\sigma .
$$

The proof is non-trivial and relies on representations of temperature functions by Fourier transforms. The role of the integrability condition (18) is clear from the point of view of the calculations but not in any intuitive way. We will show that Theorem 4 and in fact a stronger version of it, follow easily from Theorem 3 if we take the Appell cylinder point of view. 
THEOREM 5. A necessary and sufficient condition that $u(x, t) \in H^{*}(\sigma,-\sigma)$ is that

$$
u(x, t)=\sum_{n=0}^{\infty} b_{n} w_{n}(x, t), \quad|t|>\sigma
$$

where the convergence is pointwise. The coefficients $b_{n}$ are given by (20).

ProOF. We first assume that $u(x, t) \in H^{*}(\sigma,-\sigma)$. Then by Theorem $1 \mathscr{A} u(x, t) \in$ $H^{*}(-1 / \sigma, 1 / \sigma)$ so that by Theorem 3 ,

$$
\mathscr{A} u(x, \tau)=\sum_{n=0}^{\infty} a_{n} v_{n}(x, \tau), \quad|\tau|<\frac{1}{\sigma} .
$$

For $0<\tau<1 / \sigma$, we have on appealing to Theorem 2 and changing variables,

$$
\begin{aligned}
a_{n} & =\frac{1}{n ! 2^{n}} \int_{R} \mathscr{A} u(y,-\tau) w_{n}(y, \tau) d y \\
& =\frac{1}{n ! 2^{n}} \frac{1}{4 \pi i} \int_{R} u\left(-y, \frac{1}{\tau}\right) v_{n}\left(y, \frac{-1}{\tau}\right) d y \\
& =\frac{(-1)^{n}}{n ! 2^{n}} \frac{1}{4 \pi i} \int_{R} u(y, t) v_{n}(y,-t) d y
\end{aligned}
$$

where $t=1 / \tau$ satisfies $t>\sigma$. Clearly, (22) implies that

$$
\mathscr{A}^{2} u(x, t)=k(x, t) \sum_{n=0}^{\infty} a_{n} v_{n}\left(\frac{x}{t}, \frac{-1}{t}\right)
$$

will converge pointwise for $|t|>\sigma$. Therefore, since

$$
\mathscr{A}^{2} u(x, t)=k(x, t) \mathscr{A} u\left(\frac{x}{t}, \frac{-1}{t}\right)=k(x, t) k\left(\frac{x}{t}, \frac{-1}{t}\right) u\left(\frac{x / t}{-1 / t}, \frac{-1}{-1 / t}\right)=\frac{1}{4 \pi i} u(-x, t)
$$

we have

$$
u(x, t)=4 \pi i \sum_{n=0}^{\infty} a_{n} w_{n}(-x, t)=\sum_{n=0}^{\infty} b_{n} w_{n}(x, t)
$$

where $b_{n}=4 \pi i(-1)^{n} a_{n}$ are the coefficients in (20).

Conversely, if $u(x, t)$ has an expansion (21), then $\mathscr{A} u(x, t)$ has an expansion (22). Therefore $\mathscr{A} u(x, t) \in H^{*}(-1 / \sigma, 1 / \sigma)$ so that by Theorem $1, u(x, t) \in H^{*}(\sigma,-\sigma)$. This completes the proof.

THEOREM 6. A necessary and sufficient condition that $u(x, t) \in H^{*}(\sigma, \infty)$ with integrability condition (18) is that $u(x, t)$ can be extended to $U(x, t) \in H^{*}(\sigma,-\sigma)$. 
Proof. Assume first that $u(x, t)$ can be extended to $U(x, t) \in H^{*}(\sigma,-\sigma)$. Then for $t>\sigma$ :

$$
U(x,-t)=\int_{R} k(x-y,-2 t) u(y, t) d y .
$$

Therefore, since the integral converges absolutely, (see [2]), we obtain (18).

Conversely, if $u(x, t) \in H^{*}(\sigma, \infty)$ with integrability condition (18), then for all $\tau \leq-t^{\prime}$ we have

$$
\int_{R} k\left(x-y, \tau-t^{\prime}\right)\left|u\left(y, t^{\prime}\right)\right| d y<\infty .
$$

For $t>t^{\prime}>\sigma$ we have

$$
\begin{aligned}
\int_{R} k\left(x-z, \tau-t^{\prime}\right) u\left(z, t^{\prime}\right) d z & =\int_{R}\left(\int_{R} k(x-y, \tau-t) k\left(y-z, t-t^{\prime}\right) d y\right) u\left(z, t^{\prime}\right) d z \\
& =\int_{R} k(x-y, \tau-t) \int_{R} k\left(y-z, t-t^{\prime}\right) u\left(z, t^{\prime}\right) d z d y \\
& =\int_{R} k(x-y, \tau-t) u(y, t) d y
\end{aligned}
$$

so that

$$
\int_{R} k(x-y, \tau-t) u(y, t) d y
$$

converges for all $\tau<-t^{\prime}$. Since (23) is independent of $t^{\prime}$, the integral converges for all $\tau<-\sigma$, and hence by a theorem in [2] converges absolutely.

Therefore, we may define $U(x, t)=u(x, t)$ for $t>\sigma$ and

$$
U(x, t)=\int_{R} k(x-y, 2 t) u(y,-t) d y, \quad t<-\sigma .
$$

We then have $U(x, t) \in H^{*}(\sigma,-\sigma)$, and the proof is complete.

The strong integrability condition (18) enables us to extend $u(x, t)$ to larger time intervals. This situation is not unique to intervals of the second kind. A simple calculation shows:

LEMMA 7. A necessary and sufficient condition that

$$
\int_{R}|u(x, t)| e^{x^{2} / 8 t} d x<\infty, \quad t>\sigma>0
$$

is that

$$
\int_{R}|\mathscr{A} u(x, \tau)| e^{x^{2} / 8 \tau} d x<\infty, \quad-1 / \sigma<\tau<0
$$


THEOREM 8. A necessary and sufficient condition that $u(x, t) \in H^{*}(-\sigma, 0)$ and that

$$
\int_{R}|u(x, t)| e^{x^{2} / 8 t} d x<\infty, \quad-\sigma<t<0
$$

is that $u(x, t)$ can be extended to a function $U(x, t) \in H^{*}(-\sigma, \sigma)$.

PROOF. This follows easily from Theorem 6 and Lemma 7.

The relationship between the $\left\{v_{n}\right\}$ and the Hermite polynomials enabled Rosenbloom and Widder to prove:

THEOREM 9. (Rosenbloom and Widder [2]) If $u(x, t) \in H^{*}([-\sigma, 0))$ and if

$$
\int_{R}|u(x,-\sigma)|^{2} k(x, \sigma) d x<\infty,
$$

then for $0<t \leq \sigma$,

$$
\lim _{N \rightarrow \infty} \int_{R}\left|u(x,-t)-\sum_{n=0}^{N} a_{n} v_{n}(x,-t)\right|^{2} k(x, t) d x=0,
$$

$$
\int_{R}|u(x,-t)|^{2} k(x, t) d x=\sum_{n=0}^{\infty} n !(2 t)^{n}\left|a_{n}\right|^{2}
$$

where $a_{n}$ are as in (17).

We will show that the corresponding theorem for the $w_{n}$ 's follows easily from Theorem 9 using the Appell cylinder point of view.

THEOREM 10. (Rosenbloom and Widder [2]) If $u(x, t) \in H^{*}([\sigma, \infty))$ and if

$$
\int_{R} \frac{|u(x, \sigma)|^{2}}{k(x, \sigma)} d x<\infty
$$

then for $t \geq \sigma$,

$$
\begin{gathered}
\lim _{N \rightarrow \infty} \int_{R} \frac{\left|u(x, t)-\sum_{n=0}^{N} b_{n} w_{n}(x, t)\right|^{2}}{k(x, t)} d x=0 \\
\int_{R} \frac{|u(x, t)|^{2}}{k(x, t)} d x=\sum_{n=0}^{\infty} n !\left(\frac{2}{t}\right)^{n}\left|b_{n}\right|^{2}
\end{gathered}
$$

where $b_{n}$ are the coefficients in (20). 
ProOF. It is easy to see for $\tau=1 / t$ that

$$
\int_{R}|\mathscr{A} u(x,-t)|^{2} k(x, t) d x=\frac{1}{(4 \pi)^{2}} \int_{R} \frac{|u(x, \tau)|^{2}}{k(x, \tau)} d x
$$

whenever either integral is finite.

Since $u(x, t) \in H^{*}(\sigma, \infty)$, we have $\mathscr{A} u(x, t) \in H^{*}(-1 / \sigma, 0)$ and

$$
\int_{R}\left|\mathscr{A} u\left(x,-\frac{1}{\sigma}\right)\right|^{2} k\left(x, \frac{1}{\sigma}\right) d x=\frac{1}{(4 \pi)^{2}} \int_{R} \frac{|u(x, \sigma)|^{2}}{k(x, \sigma)} d x<\infty .
$$

Therefore, by Theorem 9 , for $0<t \leq 1 / \sigma$ and $\tau=1 / t$,

$$
\begin{aligned}
& \frac{1}{(4 \pi)^{2}} \int_{R} \frac{\left|u(x, \tau)-\sum_{n=0}^{N} b_{n} w_{n}(x, \tau)\right|^{2}}{k(x, \tau)} d x \\
& \quad=\int_{R}\left|\mathscr{A} u(x,-t)-\sum_{n=0}^{N} b_{n} \mathscr{A} w_{n}(x,-t)\right|^{2} k(x, t) d x \\
& \quad=\int_{R}\left|\mathscr{A} u(x,-t)-\sum_{n=0}^{N} \frac{b_{n}}{4 \pi i}(-1)^{n} v_{n}(x,-t)\right|^{2} k(x, t) d x
\end{aligned}
$$

converges to zero as $n \rightarrow \infty$ provided $b_{n}$ is the coefficient in (20). Furthermore, applying Theorem 9 to the Appell transform of $u$ we have

$$
\int_{R} \frac{|u(x, \tau)|^{2}}{k(x, \tau)} d x=\sum_{n=0}^{\infty} n !\left(\frac{2}{\tau}\right)^{n}\left|b_{n}\right|^{2}
$$

and the theorem is proved.

\section{References}

[1] P. Appell, 'Sur l'équation $\partial^{2} z / \partial x^{2}-\partial z / \partial y$ et la théorie de la chaleur', J. Math. Pures Appl. 8 (1892), 186-216.

[2] P. C. Rosenbloom and D. V. Widder, 'Expansions in terms of heat polynomials and associated functions', Trans. Amer. Math. Soc. 92 (1959), 220-266.

[3] D. V. Widder, The heat equation (Academic Press, San Diego, 1975).

University of Illinois at Chicago

Chicago, Illinois 60680

USA 